\title{
The Role Of Vitamin D In Otolaryngological Diseases: Myth Or Truth
}

\author{
Ali Bayram ${ }^{1}$, MD \\ ${ }^{1}$ Department of ENT, Kayseri City Hospital, Kayseri, Turkey
}

\begin{abstract}
Inthepresentreview, weaimedtoevaluatetheroleofvitamin Dinotolaryngologicaldiseases.Sincetherecognitionofvitamin Dasasteroidhormone, ithasattractedcliniciansasa research issue due to its unique and complicated functions in human bodyprocesses. Numerousstudieshaveinvestigated theassociationbetweenvitaminDandhumandiseases, includinginthe otolaryngologicalfield. However, althoughvitaminDalterations
\end{abstract}

maytheoreticallybe involved in the pathogenesis of several otolaryngological diseases, a clear association has not been demonstrated dueto inconsistentresultsfrom thestudiesto date.Furtherrandomizedcontrolled trialswithlargepatient populationsarerequiredtodeterminetheexactroleofvitamin $D$ in otolaryngological diseases.

Keywords: Vitamin D, otorhinolaryngologic diseases, immunity
Vitamin D is an essential nutrient that has a well-known regulatory function in calcium and phosphate metabolism. Recently, vitamin D has been recognized as a steroid hormone with the identification of vitamin D receptors in many tissues, such as lymphocytes, kidney, ovaries, stomach, thymus, pancreas, skin and parathyroid glands. ${ }^{[1]}$ In relation to recent discoveries regarding the vitamin $\mathrm{D}$ receptor, researchers have determined new functions for vitamin D including immunoregulation, induction of cell differentiation and control of other hormonal systems ${ }^{[2]}$, and vitamin $\mathrm{D}$ has now been accepted as a member of a complex endocrine pathway termed the 'vitamin D endocrine system'. ${ }^{[3]}$ Since the recognition of vitamin $\mathrm{D}$ as a steroid hormone, it has attracted clinicians due to its unique and complicated functions regarding human body processes. Numerous studies have investigated the association between vitamin $\mathrm{D}$ and human diseases including in the otolaryngological field. In the present review, we aimed to evaluate the role of vitamin $\mathrm{D}$ in otolaryngological diseases.

\section{Vitamin D Physiology and Metabolism}

The vitamin $\mathrm{D}$ endocrine system contains three forms of vitamin D; cholecalciferol, calcidiol (25-hydroxyvitamin D) and calcitriol (1,25-dihydroxyvitamin D). Cholecalciferol is the natural form of vitamin D. It can be naturally synthesized in the skin through sunlight exposure or can be supplemented by dietary intake. ${ }^{[4]}$ Calcidiol is produced from cholecalciferol as a prehormone in the liver, which is generally accepted as a blood indicator of vitamin D status. Calcidiol is converted into calcitriol both in the kidneys and other tissues by enzymatic hydroxylation $(25(\mathrm{OH})$ D3-1-hydroxylase and 25(OH)D3-24-hydroxylase). Calcitriol, as the physiologically active form of vitamin D, increases intestinal absorption of calcium and phosphorus, and promotes bone resorption in concert with parathyroid hormone. ${ }^{[2,5]}$

\section{Vitamin D and Immunoregulation}

The immunoregulatory functions of vitamin $\mathrm{D}$ have been known for more than 30 years. ${ }^{[6]}$ Vitamin D directly regu-
Correspondence: Ali Bayram, MD, Kayseri City Hospital, Şeker Mah. Molu Village, Kayseri 38080, Turkey E-mail: dralibayram@gmail.com

Received: May 30, 2018; Accepted: June 25, 2018
Online available at: www.entupdates.or DOI:10.32448/entupdates.459029 
lates B cell, T cell, dendritic cell, macrophage and monocyte functions. ${ }^{[7,8]}$ Vitamin D suppresses T-cell proliferation ${ }^{[9,10]}$, plasma cell differentiation and immunoglobulin secretion ${ }^{[11]}$, including IgE. ${ }^{[12]}$ Vitamin D also induces apoptosis of activated B cells and the switch from Th1 to Th2. ${ }^{[13]}$

Vitamin D also has numerous effects on the innate immune system. Vitamin D decreases the immunostimulatory capacity of dendritic cells and provides them tolerogenic properties. ${ }^{[14]}$ It increases the antimicrobial activity of freshly isolated monocytes and decreases T-cell stimulation and macrophage inflammation. ${ }^{[15]}$ Chemotactic and phagocytic effects of monocytes and macrophages can be increased by vitamin $\mathrm{D}$. Also, an increment in the synthesis of antimicrobial peptides, defensins and cathelicidin can be presented from natural killer cells and respiratory tract epithelial cells in an enriched active vitamin D environment. ${ }^{[15]}$ In addition, vitamin D causes the upregulation of calprotectin and S100 protein levels, which contribute significantly to the functions of the natural immune system. ${ }^{[16]}$ Vitamin D also has modulatory functions on a variety of cytokines that are considered to have an active role in the pathogenesis of many autoimmune diseases. In most studies, it is indicated that Th1-related cytokines such as interferon- $\gamma$, tumor necrosis factor- $\alpha$ and IL-2 are generally inhibited by vitamin $\mathrm{D}$, whereas Th2-related cytokines including IL-4 and IL-10 are upregulated by vitamin D. ${ }^{[13]}$ In conclusion, vitamin D has several modulatory functions in the immune system that generate a positive correlation between the immune system and vitamin D status. Vitamin $\mathrm{D}$ deficiency causes impaired immune responses that lead to increased infection rates including in the upper respiratory tract. ${ }^{[15]} \mathrm{Also}$, vitamin $\mathrm{D}$ status has been investigated in recent studies concerning allergic and autoimmune pathologies including otolarygological diseases due to its immunoregulatory effects.

\section{Vitamin D and Otolaryngological Diseases}

A high incidence of vitamin D deficiency has been reported in patients attending otolaryngology clinics in different studies. Bartley et al ${ }^{[17]}$ investigated plasma vitamin D levels in 48 patients who attended a general otolaryngology clinic and reported that $2 \%$ had a level of $17.5 \mathrm{nmol} / \mathrm{L}$ or less, $58 \%$ below $50 \mathrm{nmol} / \mathrm{L}$ and all were below $80 \mathrm{nmo-}$ 1/L. Similarly, Taneja and Taneja ${ }^{[18]}$ detected vitamin D deficiency in 83 out of 86 patients and concluded that the incidence of vitamin D deficiency is extremely common in patients visiting outpatient otolaryngology clinics. Recently, the association between otolaryngological diseases (i.e allergic rhinitis, otitis media, obstructive sleep apnea, etc.) and vitamin $\mathrm{D}$ status has gained a marked interest among clinicians and several reports have been introduced to the literature.

The role of vitamin $\mathrm{D}$ in allergic rhinitis (AR) is still a matter of debate, although several studies have investigated a possible relationship. A shift from a Th1 to Th2 phenotype in the proliferation of CD4+ $\mathrm{T}$ cells is a possible contributing factor in the pathogenesis of AR. In a similar manner, vitamin D induces a switch from Th1 to Th2 by enhancing the development of Th2 cells and suppresses the differentiation and functions of Th17 cells, which have an important role in the disease course of AR. ${ }^{[19]}$ Similarities between the pathogenesis of $A R$ and vitamin $D$ functions may suggest a role of vitamin $\mathrm{D}$ in the development of $\mathrm{AR}$, although conflicting data have been acquired through epidemiological and clinical studies. Mai et al ${ }^{[20]}$ investigated the role of low vitamin D status in the development of AR in a large population-based health study and reported that low vitamin D status was related to an increased risk of $\mathrm{AR}$ in men and a seemingly reduced risk in women. On the contrary, in the study of Wjst and Hyppönen ${ }^{[21]}$, including 18,224 adults, they found that AR prevalence was increased with levels of vitamin D. A recent systematic review and meta-analysis investigating vitamin D status, aeroallergen sensitization and AR revealed a significant age- and sex-specific relationship between vitamin D status and the risk of aeroallergen sensitization and AR. ${ }^{[22]}$ The meta-analysis indicated that children with serum vitamin D levels lower than $50 \mathrm{nmol} / \mathrm{L}$ had an increased risk of aeroallergen sensitization. Also, vitamin $\mathrm{D}$ deficient men, but not women, were found to have an increased risk of AR according to the meta-analysis. Further studies conducted with large patient populations are required to clarify the exact mechanism of vitamin $\mathrm{D}$ in the pathogenesis of $\mathrm{AR}$ and the clinical implications of vitamin D supplementation on the risk of AR occurrence.

Due to its immunoregulatory functions, vitamin D has significant roles in improvement of immune function and reduction of inflammation. [23] Therefore, vitamin D deficiency may be associated with respiratory tract infections (RTI) including acute otitis media, pharyngotonsillitis, rhinosinusitis, bronchiolitis and pneumonia, especially in children. ${ }^{[2,25]}$ Available data support a role of vitamin D deficiency in the risk of pediatric tuberculosis, recurrent 
acute otitis media, and severe bronchiolitis, while further studies are required to clarify a relation in children with recurrent pharyngotonsillitis, acute rhinosinusitis and community-acquired pneumonia. However, the data are insufficient to allow definitive conclusions regarding a supplementation regimen for children. ${ }^{[23]}$ A systematic review of clinical studies including all ages determined a statistically significant relationship between low vitamin D status and increased risk of both upper and lower RTI, although vitamin $\mathrm{D}$ supplementation trials did not reveal consistent protective effects against RTI.[26] Further studies are required especially to determine an optimal vitamin D supplementation regimen depending on the type of RTI.

Obstructive sleep apnea (OSA) is a serious disease that is characterized by apnea and hypopnea events during sleep. The severity of the disease depends on the number of apnea and hypopnea events per hour and particularly those with moderate and severe OSA have an increased risk of cardiovascular morbidities. ${ }^{[27]}$ Currently, several studies have investigated any association between vitamin $\mathrm{D}$ and OSA. Upala and Sanguankeo ${ }^{[28]}$ published the first systematic review and meta-analysis revealing the presence of lower vitamin D levels in patients with OSA compared to controls. In a more recent systematic review and meta-analysis, Neighbors et al ${ }^{[29]}$ reported that a relative insufficiency in serum vitamin D levels was present in OSA patients compared to controls, which was incrementally exacerbated with increasing severity of sleep apnea. On the other hand, the underlying mechanism of a vitamin D and OSA association is less clear than its presentation. One possible explanation is that low vitamin D levels arise due to a hypoxia-induced mechanism.[30] Vitamin D insufficiency has been shown in obese patients regardless of OSA status. ${ }^{[31]}$ Since higher body mass index is a common morbidity in OSA, lower levels of vitamin D in OSA patients may be associated with obesity rather than OSA. ${ }^{[29]}$ In conclusion, further randomized controlled studies are needed to evaluate the association between OSA, vitamin D and obesity for a better understanding of underlying mechanisms.

Vitamin D related calcium channel proteins in the epithe- lium are known to be involved in calcium metabolism of the vestibular organ. Vitamin D has a significant role in the development and maintenance of otoconia and proper otolith function. ${ }^{[32]}$ Decreased bone mineral density was shown in patients with benign paroxysmal positional vertigo (BPPV) in the literature. Vibert et al ${ }^{[33]}$ reported that the ratio of osteoporosis was higher among BPPV patients than controls and revealed an association between calcium metabolism disorders and the occurrence of BPPV. Since vitamin $\mathrm{D}$ has significant functions in maintaining proper bone structure and calcium metabolism, alterations of vitamin $\mathrm{D}$ levels may be seen in BPPV. In a retrospective study including $232 \mathrm{BPPV}$ patients, vitamin D was suggested as a recurrence factor for BPPV, regardless of age, gender, follow-up period and type of BPPV.[34] In severe vitamin $\mathrm{D}$ deficiencies, improvement of serum vitamin $\mathrm{D}$ levels was shown to diminish the recurrence rate of BPPV. ${ }^{[32]}$ On the contrary, Maslovara et al ${ }^{[35]}$ indicated the need for supplemental therapy due to a low level of serum vitamin D3 in most BPPV patients participating in their study, although no significant difference was shown in vitamin D3 levels in patients with and without recurrence. Future studies should be focused on clarifying the effect of vitamin D supplementation in decreasing recurrence rates in patients with recurrent BPPV.

\section{Conclusion}

Vitamin D attracts clinicians due its unique and complicated functions regarding human body processes. Numerous studies have investigated a possible association between vitamin D and otolaryngological diseases. Due to the inconsistency among the relevant studies, vitamin $\mathrm{D}$ seems to be an ongoing issue for further research in the otolaryngological field. Although further randomized controlled studies with large patient populations are required to demonstrate the clinical benefits, supplementation of vitamin D in deficient patients may have clinical implications for the course of otolaryngological disease. 


\section{References}

1. Abuzeid WM, Akbar NA, Zacharek MA. Vitamin D and chronic rhinitis. Curr Opin Allergy Clin Immunol 2012;12:13-7.

2. Akbar NA, Zacharek MA. Vitamin D: immunomodulation of asthma, allergic rhinitis, and chronic rhinosinusitis. Curr Opin Otolaryngol Head Neck Surg 2011;19:224-8.

3. Norman A. From vitamin D to hormone D: fundamentals of the vitamin D endocrine system essential for good health. Am J Clin Nutr 2008;88:491-9.

4. Deluca HF. The metabolism, physiology, and function of vitamin D. In: Kumar R, editor. Vitamin D: Basic and Clinical Aspects. Boston: Martinus Nijhoff Publishers; 1984. p. 1-68.

5. Kumar R. The metabolism and mechanism of action of 1,25-dihydroxyvitamin D3. Kidney Int 1986;30:793-803.

6. Tsoukas CD, Provvedini DM, Manolagas SC. 1,25-dihydroxyvitamin D3: a novel immunoregulatory hormone. Science 1984;224:1438-40.

7. Mulligan JK, Bleier BS, O'Connell B, et al. Vitamin D3 correlates inversely with systemic dendritic cell numbers and bone erosion in chronic rhinosinusitis with nasal polyps and allergic fungal rhinosinusitis. Clin Exp Immunol 2011;164:312-20.

8. Wolff AE, Jones AN, Hansen KE. Vitamin D and musculoskeletal health. Nat Clin Pract Rheumatol 2008;4:580-8.

9. Rigby WF, Stacy T, Fanger MW. Inhibition of T lymphocyte mitogenesis by 1,25-dihydroxyvitamin D3 (calcitriol). J Clin Invest 1984;74:1451-5.

10. Cantorna MT, Waddell A. The vitamin D receptor turns off chronically activated T cells. Ann N Y Acad Sci 2014;1317:70-5.

11. Chen S, Sims GP, Chen XX, Gu YY, Chen S, Lipsky PE. Modulatory effects of 1,25-dihydroxyvitamin D3 on human B cell differentiation. J Immunol 2007;179:1634-47.

12. Heine G, Anton K, Henz BM, Worm M. 1alpha,25-dihydroxyvitamin D3 inhibits anti-CD40 plus IL-4-mediated IgE production in vitro. Eur J Immunol 2002;32:3395-404.

13. Tian HQ, Cheng L. The role of vitamin D in allergic rhinitis. Asia Pac Allergy 2017;7:65-73

14. Kamen DL, Tangpricha V. Vitamin D and molecular actions on the immune system: modulation of innate and autoimmunity. J Mol Med (Berl) 2010;88:441-50.

15. Akcan FA, Dündar Y, Akcan HB, et al. Clinical role of vitamin D in prognosis of otitis media with effusion. Int J Pediatr Otorhinolaryngol 2018;105:1-5.

16. Szodoray P, Nakken B, Gaal J, et al. The complex role of vitamin D in autoimmune diseases. Scand J Immunol 2008;68:261-9.

17. Bartley J, Reid D, Morton RP. Prevalence of vitamin D deficiency among patients attending a general otolaryngology clinic in South Auckland. Ann Otol Rhinol Laryngol 2009;118:326-8.

18. Taneja MK, Taneja V. Vitamin D deficiency in E.N.T. patients. Indian J Otolaryngol Head Neck Surg 2013;65:57-60.

19. Osguthorpe JD. Pathophysiology of and potential new therapies for allergic rhinitis. Int Forum Allergy Rhinol 2013;3:384-92.
20. Mai XM, Chen Y, Camargo CA Jr, Langhammer A. Serum 25-hydroxyvitamin D levels and self-reported allergic rhinitis in Norwegian adultsThe HUNT Study. Allergy 2014;69:488-93.

21. Wjst M, Hyppönen E. Vitamin D serum levels and allergic rhinitis. Allergy 2007;62:1085-6.

22. Aryan Z, Rezaei N, Camargo CA Jr. Vitamin D status, aeroallergen sensitization, and allergic rhinitis: A systematic review and meta-analysis. Int Rev Immunol 2017;36:41-53.

23. Esposito S, Lelii M. Vitamin D and respiratory tract infections in childhood. BMC Infect Dis 2015;15:487.

24. Esposito S, Baggi E, Bianchini S, Marchisio P, Principi N. Role of vitamin D in children with respiratory tract infections. Int J Immunopathol Pharmacol 2013;26:1-13.

25. Marchisio P, Nazzari E, Torretta S, Esposito S, Principi N. Medical prevention of recurrent acute otitis media: an updated overview. Expert Rev Anti Infect Ther 2014;12:611-20.

26. Jolliffe DA, Griffiths CJ, Martineau AR. Vitamin D in the prevention of acute respiratory infection: systematic review of clinical studies. J Steroid Biochem Mol Biol 2013;136:321-9.

27. Punjabi NM. The epidemiology of adult obstructive sleep apnea. Proc Am Thorac Soc 2008;5:136-43.

28. Upala S, Sanguankeo A. Association between 25-Hydroxyvitamin D and Obstructive Sleep Apnea: A Systematic Review and Meta-Analysis. J Clin Sleep Med 2015;11:1347.

29. Neighbors CLP, Noller MW, Song SA, et al. Vitamin D and obstructive sleep apnea: a systematic review and meta-analysis. Sleep Med 2018;43:100-8.

30. Persson LJ, Aanerud M, Hiemstra PS, et al. Chronic obstructive pulmonary disease is associated with low levels of vitamin D. PLos One 2012; 7:e38934.

31. Grace C, Vincent R, Aylwin SJ. High prevalence of vitamin D insufficiency in a United Kingdom urban morbidly obese population: implications for testing and treatment. Surg Obes Relat Dis 2014;10:355-60.

32. Talaat HS, Kabel AM, Khaliel LH, Abuhadied G, El-Naga HA, Talaat AS. Reduction of recurrence rate of benign paroxysmal positional vertigo by treatment of severe vitamin D deficiency. Auris Nasus Larynx 2016;43:237-41.

33. Vibert D, Kompis M, Hausler R. Benign paroxysmal positional vertigo in older women may be related to osteoporosis and osteopenia. Ann Otol Rhinol Laryngol 2003;112;885-9.

34. Rhim GI. Serum vitamin D and recurrent benign paroxysmal positional vertigo. Laryngoscope Investig Otolaryngol 2016;1:150-3.

35. Maslovara S, Butkovic Soldo S, Sestak A, Milinkovic K, Rogic-Namacinski J, Soldo A. $25(\mathrm{OH}) \mathrm{D} 3$ levels, incidence and recurrence of different clinical forms of benign paroxysmal positional vertigo. Braz J Otorhinolaryngol 2018;84:453-9.

This is an open access article distributed under the terms of the Creative Commons Attribution-NonCommercial-NoDerivs 3.0 Unported (CC BYNC-ND3.0) Licence (http://creativecommons.org/licenses/by-nc-nd/3.0/) which permits unrestricted noncommercial use, distribution, and reproduction in any medium, provided the original work is properly cited.

Please cite this article as: Bayram A. The role of vitamin D in otolaryngological diseases: myth or truth. ENT Updates 2018;8(2): 120-123. 